\title{
Evaluación de avena hidropónica (Arrenatherium elatius) en la alimentación de conejos
}

\author{
en la etapa de engorde
}

\section{Evaluation of hydroponic oats (Arrenatherium elatius) in the feeding of rabbits in the fattening stage \\ Núñez-Torres Oscar Patricio*, Lozada-Salcedo Euclides Efraín, Rosero-Peñaherrera Marco Antonio, Cruz-Tobar Eduardo Saúl, Aragadvay-Yungan Ramon Gonzalo}

\begin{tabular}{|c|}
\hline Datos del Artículo \\
\hline $\begin{array}{l}\text { Universidad Técnica de Ambato. } \\
\text { Facultad de Ciencias Agropecuarias. } \\
\text { Cantón Cevallos. } \\
\text { Tungurahua - Ecuador. } \\
\text { Casilla postal: 18-01-334. } \\
\text { Telf: }(+593) 032872630-0985471191\end{array}$ \\
\hline $\begin{array}{l}\frac{\text { ee.lozada@uta.edu.ec }}{\text { ma.rosero@uta.edu.ec }} \\
\text { rg.aragadvay@uta.edu.ec }\end{array}$ \\
\hline *Dirección de contacto: \\
\hline $\begin{array}{l}\text { Universidad Técnica de Ambato. } \\
\text { Facultad de Ciencias Agropecuarias. } \\
\text { Cantón Cevallos. Tungurahua } \\
\text { Ecuador. Casilla postal: 18-01-334. } \\
\text { Telf: }(+593) 032872630-0985471191\end{array}$ \\
\hline $\begin{array}{l}\text { Oscar Patricio Núñez-Torres. } \\
\text { E-mail address : op.nunez@uta.edu.ec }\end{array}$ \\
\hline Palabras clave: \\
\hline $\begin{array}{l}\text { Forraje, } \\
\text { hidropónico, } \\
\text { avena. }\end{array}$ \\
\hline
\end{tabular}

J. Selva Andina Anim. Sci. 2017; 4(1):59-71.

\section{Historial del artículo.}

Recibido mayo, 2016.

Devuelto diciembre 2016

Aceptado febrero, 201

Disponible en línea, abril, 2017

Editado por:

Selva Andina

Research Society
(C) 2017. Journal of the Selva Andina Animal Science. Bolivia. Todos los derechos reservados.

La presente investigación se realizó en el sector de La Concepción, perteneciente a la parroquia Atahualpa, cantón Ambato, provincia de Tungurahua, tiene como fin evaluar la producción de forraje verde hidropónico de avena, su costo y su influencia en el mejoramiento de la ganancia de peso. Para este trabajo se contó con 21 animales machos de raza neozelandés con un peso promedio de $1179 \mathrm{~g}$, de 8 semanas de edad conformados por tres tratamientos T1 (forraje verde hidropónico de avena sin solución nutritiva), T2 (forraje verde hidropónico de avena con solución nutritiva y T0 (avena de corte), cada tratamiento consta de 7 repeticiones, la unidad experimental fue de un animal en estudio. El forraje verde hidropónico de avena fue elaborado dentro de un invernadero de $18 \mathrm{~m}^{2}$ cubierto internamente con tela de sombra negra al $65 \%$ y realizando una serie de procedimientos como: selección, lavado, pre-germinación, germinación, siembra de la semilla en las bandejas de plástico y riego de la siembra con solución nutritiva y agua, para finalizar con la cosecha realizando al día 12 para ser consumido por los conejos. Se aplicó el diseño completamente al azar, para la interpretación de los resultados se realizaron análisis de varianza y prueba de Tukey 5\%. Obteniendo los mejores resultandos con T2 con ganancia de peso diario de $35.09 \mathrm{~g}$; mejoría en el índice de conversión alimenticia con 5.5, peso promedio del animal al mercado $3136.6 \mathrm{~g}$; 0 \% de mortalidad, una buena rentabilidad con un beneficio costo de USD1.34.

\section{Abstract}

The present research was carried out in the sector of La Concepción, belonging to the Atahualpa parish, Ambato canton, province of Tungurahua, to evaluate the production of green oat hydroponic forage, its cost and its influence on the improvement of the weight. For this work, 21 male New Zealand breed animals with an average weight of $1179 \mathrm{~g}$, of 8 weeks of age were composed of three treatments T1 (hydroponic green forage of oats without nutritive solution), T2 (green forage hydroponic of oats with solution nutrient and T0, each treatment consists of 7 replicates, the experimental unit was an animal under study. The hydroponic green forage of oats was elaborated inside an $18 \mathrm{~m}^{2}$ greenhouse covered internally with black shade cloth at $65 \%$ And carrying out a series of procedures such as: selection, washing, pregermination, germination, planting of the seed in the plastic trays and irrigation of the sowing with nutritive solution and water, to finish with the harvest making at day 12 to be The results were analyzed by variance and Tukey $5 \%$ test, obtaining the best results with T2 with a daily weight gain of $35.09 \mathrm{~g}$; Improvement in feed conversion rate with 5.5, Average weight of the animal to the market $3136.6 \mathrm{~g}, 0 \%$ mortality, good profitability with a cost benefit of USD1.34.

\section{Key words:}




\section{Introducción}

En la provincia de Tungurahua últimamente existen variaciones climáticas demasiado bruscas las que limitan la producción de pastos de calidad que aporten con nutrientes necesarios para animales. La búsqueda de soluciones a este problema, el sistema de producción de forraje verde hidropónico $(\mathrm{FVH})$ representa una alternativa no convencional, importante para la obtención de forrajes de muy buena calidad nutricional a través del año, utilizando pequeñas superficies de terreno (Rodriguez-Muela et al. 2012, Cerrillo-Soto et al. 2012, Fuentes et al 2011, sugieren que para la producción de forraje verde hidropónico de avena (FVHA) la densidad de siembra óptima de $5.0-6.4 \mathrm{~kg} / \mathrm{m}^{2}$. Este forraje se ha incorporado en distintos sistemas de producción animal, con el fin de reemplazar parcial o totalmente algunos componentes de la ración diaria (Morales et al. 2009), siendo este último importante, ya que los costos de alimentación de conejos a base de concentrados comerciales (CC) resulta elevada (Palma \& Hurtado 2010).

El FVH resulta el producto de la germinación de granos de cereales como avena, cebada, maíz, arroz, trigo y sorgo, estando listos para la cosecha en periodos comprendidos entre los 9 a 15 días posteriores a la siembra, en la que se aprovecha la energía solar, la solución de nutrientes minerales, permite incrementar la producción de biomasa por $\mathrm{m}^{2}$, mejorar la calidad nutricional y optimizar el tiempo de la cosecha del forraje (Morales et al. 2009). CerrilloSoto et al. (2012) han reportado una producción de biomasa de $13.0 \mathrm{~kg}$ FVHA $/ \mathrm{m}^{2}$ con calidades nutricionales en proteína $15.6 \%$ de materia seca (MS), contenido de energía metabolizable $(2.5 \mathrm{Mcal} / \mathrm{Kg}$ /MS) y contenido de proteína metabolizable (73 g $/ \mathrm{Kg} / \mathrm{MS})$.
Por su parte, Fuentes et al. (2011), sugieren no postergar la cosecha por periodos muy largos de tiempo ya que disminuye su calidad, por tal motivo recomiendan realizar dicha actividad a los 10 días, además indican que al cumplir con esta condición se obtienen forrajes de muy buena calidad nutricional con un contenido del $36.86 \%$ MS, $14.79 \%$ de proteína bruta (PB) y $18.77 \%$ de fibra cruda (FC). Por tanto, Fuentes-Carmona et al. (2011) recomiendan remplazar hasta un $50 \%$ de la dieta a base de CC con FVHA ya que esta cantidad no afecta al consumo de alimento, tiempo de peso vivo al sacrificio, peso vivo final y rendimiento a la canal de conejos en la etapa de engorde dadas, por la alta palatabilidad, digestibilidad, contenidos óptimos de energía, minerales y vitaminas.

Estudios realizados en la Evaluación de cuatro forrajes hidropónicos en la alimentación de cuyes (Cavia porcellus), durante la fase de crecimiento y engorde la variable ganancia de peso total (GPT), presento diferencias estadísticas $(\mathrm{P}<0.01)$, siendo la mayor ganancia de peso para el tratamiento T3 (forraje verde hidropónico de maíz) con 1150.7 g /cobayo. Como lo manifiesta (Rea \& Mora 2012). La construcción de un invernadero fue conseguir crecimiento rápido, saludable, económico de los cultivos, además de controlar temperaturas, humedad, luminosidad, así como evitar ataque de plagas, lograr cosechas redituales fuera de temporada. La abundancia de sol, agua, temperatura son factores prioritarios y determinantes para una muy buena o precaria cosecha. (Samperio 1999).

El objetivo de la presente investigación fue evaluar la respuesta productiva en la ganancia de peso de conejos raza neozelandés en la etapa de engorde 
alimentados con FVHA sin solución nutritiva (T1), con solución nutritiva (T2) y avena de corte (T0).

\section{Materiales y métodos}

El ensayo se realizó en la Provincia Tungurahua, cantón Ambato Parroquia Atahualpa, la temperatura promedio es de 12 a $23{ }^{\circ} \mathrm{C}$, humedad relativa $60 \%$, luminosidad $12 \mathrm{~h} /$ día, altitud $2500 \mathrm{msnm}$, longitud
W $78^{\circ} 37^{\prime} 11^{\prime \prime}$, coordenadas S $1^{\circ} 14^{\prime} 30^{\prime \prime} \mathrm{O} 78^{\circ} 37^{\prime}$ 11". Para el análisis se utilizó (DCA), diseño completamente al azar, con 3 tratamientos, 7 repeticiones. (Tabla 1). La unidad experimental (UI) consta de un animal con un total de 21 animales en la ejecución del proyecto. Todos los datos fueron puestos al cálculo de pruebas de significancia de Tukey al $5 \%$ con el análisis de varianza.

Tabla 1 Tratamientos

\begin{tabular}{cccc}
\hline Tratamiento & Simbología & Forraje Verde Hidropónico de Avena/Avena de corte & Repeticiones \\
\hline 1 & T0 & $100 \%$ avena de corte & 7 \\
2 & T1 & $100 \%$ FVH de avena sin solución nutritiva & 7 \\
3 & T2 & $100 \%$ FVH de avena con solución nutritiva & 7 \\
\hline
\end{tabular}

El invernadero fue de madera cubierta con plástico de polietileno UV6, con ventoleras en los lados. En el interior cubierto con tela de sombra negra al $65 \%$, sus medidas fueron: $6 \mathrm{~m}$ de largo $\mathrm{x} 3 \mathrm{~m}$ de ancho $\mathrm{x}$ $3 \mathrm{~m}$ de alto. Se construyó 3 jaulas de madera individuales para ubicar a los 21 conejos destetados de 8 semanas de edad (machos), en cada jaula se ubicaron 7 animales la superficie total de la jaula fue de $2.10 \mathrm{~m}^{2}$. Se registró semanalmente el peso inicial, primer dato que se tomó antes de ingresar los conejos a sus jaulas, para el consumo de alimento, se pesó el alimento utilizando, balanza analítica marca Toledo con una precisión de $0.1 \mathrm{~g}^{\circledR}$ capacidad de 5 $\mathrm{kg}$, escala de verificación $1 \mathrm{~g}$, en la mañana, se alimentaba a los conejos y por la tarde se pesó nuevamente. Se pesó el sobrante del alimento por la mañana y la tarde.

La mortalidad se registró, los animales muertos, se restaron del total de vivos, los factores en estudio son: T0 avena de corte, T1 forraje verde hidropónico de avena sin solución nutritiva. T2 forraje verde hidropónico de avena con solución nutritiva, la que debe contener rangos entre 80 y 140 ppm de nitró61 geno como nitritos y unas 10 a 15 ppm como amonio, alrededor de unas $30 \mathrm{ppm}$ de fosforo como fosfato, de 90 a $130 \mathrm{ppm}$ de potasio, de 90 a 160 ppm de calcio, 20 a 30 ppm de magnesio y 45 a 100 ppm de sulfatos. En relacion a los microelementos puede decirse que generalmente son suficientes con las impurezas que se encuentran en sales comerciales que se deben usar para preparar la fórmula base. (Leandro 2010).

La conversión alimenticia se obtuvo mediante el registro ganancia de peso de cada animal y el consumo de alimento, efectuando lecturas cada semana, Se aplicó la formula consumo de alimento/ganancia de peso, además se registró todos los gastos necesarios para la producción de conejos y el uso de forraje por tratamiento, de esta manera se estableció la diferencia entre los tratamientos mediante la relación beneficio/costo.

Para la selección de semilla, es el punto más importante en la producción del FVH, la selección del grano a utilizar depende de múltiples factores, la disponibilidad de semillas adaptadas a las condiciones del producto final, calidad germinativa, deben 
estar íntegras y que no han sido tratados con algún pesticida tóxico. (Moreno 2013). La semilla de avena fue adquirida en el Instituto de Agricultura Limpia de la Facultad de Ciencias Agropecuarias, para la pre-germinación de la avena hidropónica se pesó la semilla, para dejarla en remojo durante $24 \mathrm{~h}$, luego se lavaron las semillas, eliminando las sustancias flotantes, piedrecillas o basura que pudiesen tener, con un porcentaje de desecho del $2 \%$. Posteriormente se colocó en un recipiente con agua, se añadió hipoclorito de sodio al $1 \%$ en dosis de $1 \mathrm{~mL} / \mathrm{L}$ para desinfectar la semilla y dejar reposar durante 15 min, nuevamente se lavaron con agua y se colocaron dentro de un cedazo tapado con papel periódico durante $2 \mathrm{~h}$ bajo sombra para que estile el exceso de agua. Para la germinación de las semillas pregerminadas se colocaron dentro de una funda negra con orificios en la parte inferior, esto para que se oxigenen durante el proceso de germinación y se depositaron dentro de un balde tapado con periódico húmedo durante 3 días.

A los tres días de germinación se destapó las fundas negras encontrándose las primeras raicillas de las semillas, se procedió a sembrar en bandejas plásticas de 45 x $30 \mathrm{~cm}$ con orificios en un extremo para evitar la acumulación del agua. Se colocaron un promedio de $182 \mathrm{~g}$ de semilla por bandeja con su respectiva rotulación. Para la preparación de la solución nutritiva o fertilizante para el FVH se lo hizo en un balde utilizando las siguientes sustancias con sus dosis respectivas. Las dosis de sustancias utilizadas para la preparación de la solución nutritiva son: $\mathrm{Ca}(\mathrm{NO} 3) 2$, dosis $1.20 \mathrm{~g} / \mathrm{L}$; KNO3, dosis 0.87 $\mathrm{g} / \mathrm{L}$; (NH4) (NO3), dosis $0.54 \mathrm{~g} / \mathrm{L} ; \mathrm{H} 3 \mathrm{PO} 4,0.20$ $\mathrm{g} / \mathrm{L} ; \mathrm{MgSO} 4,0.52 \mathrm{~g} / \mathrm{L}$; micronutrientes $0.10 \mathrm{~g} / \mathrm{L}$.

\section{Resultados}

La ganancia de peso semanal de la primera a la cuarta semana (tabla 2), se reporta el análisis de varianza para la variable ganancia de peso desde la semana uno a la semana cuatro de engorde, se determinó que existen diferencias estadísticas al $1 \%$ para tratamientos en la ganancia de peso de cada tratamiento. El coeficiente de variación para la primera semana fue de $2.28 \%$, para la segunda semana de $1.25 \%$, para la tercera semana de $0.90 \%$ y para la cuarta semana de $1.44 \%$, cuyos valores demuestran una aceptable confiabilidad en los resultados. En la tercera semana se registran tres rangos de significación, siendo el mejor T2 (100\% FVH de avena con solución nutritiva) con una ganancia de peso promedio de $244.71 \mathrm{~g}$, el segundo rango lo ocupa T1 (100\% FVH de avena sin solución nutritiva) con una ganancia de peso de $239.86 \mathrm{~g}$ y el tercer rango lo ocupa T0 (Avena de corte) con una ganancia de peso de $102.00 \mathrm{~g}$. Para la cuarta semana se registran tres rangos de significación siendo el mejor T2 (100\% FVH de avena con solución nutritiva) con una ganancia de peso promedio de $264.57 \mathrm{~g}$, el segundo rango lo ocupa T1 (100\% FVH de avena sin solución nutritiva) con una ganancia de peso de $257.00 \mathrm{~g}$ y el tercer rango lo ocupa T0 (Avena de corte) con una ganancia de peso de $108.60 \mathrm{~g}$. Con estos resultados se deduce que la utilización del FVH con solución nutritiva produce mayor ganancia de peso debido a que el forraje posee un alto contenido nutricional.

Aplicando la prueba de Tukey al 5\%, para la variable ganancia de peso desde la semana uno a la semana cuatro (tabla 3), se registran tres rangos de significación en la primera semana siendo el mejor T2 (100\% FVH de avena con solución nutritiva) con una ganancia de peso promedio semanal de 201.57 
g, el segundo rango lo ocupa T1 (100\% FVH de avena sin solución nutritiva) con una ganancia de peso de 195.71g y el último rango de significación lo ocupa T0 (Avena de corte) con una ganancia de peso de 75.8 g. En la segunda semana se registran tres rangos de significación siendo el mejor T2 (100\% FVH de avena con solución nutritiva) con una ganancia de peso promedio de $218.86 \mathrm{~g}$.

Tabla 2 Análisis de varianza para la variable ganancia de peso: 1era a 4ta semana

\begin{tabular}{|c|c|c|c|c|c|c|c|c|c|}
\hline \multirow{2}{*}{ F de V } & \multirow[t]{2}{*}{ GL } & \multicolumn{2}{|c|}{ SEMANA 1} & \multicolumn{2}{|c|}{ SEMANA 2} & \multicolumn{2}{|c|}{ SEMANA 3} & \multicolumn{2}{|c|}{ SEMANA 4} \\
\hline & & $\begin{array}{c}\text { CM } \\
\end{array}$ & Valor de F & CM & Valor de F & CM & Valor de $\mathbf{F}$ & CM & Valor de F \\
\hline Tratamientos & 2 & 27858.08 & $1938.44 * *$ & 29994.47 & $5792.03 * *$ & 36294.07 & $10697.2 * *$ & 42764.38 & $4252.14 * *$ \\
\hline Error & 16 & 14.37 & & 5.18 & & 3.39 & & 10.06 & \\
\hline Total & 18 & & & & & & & & \\
\hline C.V (\%) & & \multicolumn{2}{|c|}{2.28} & \multicolumn{2}{|c|}{1.25} & \multicolumn{2}{|c|}{0.90} & \multicolumn{2}{|c|}{1.44} \\
\hline
\end{tabular}

Tabla 3 Prueba de Tukey al $5 \%$ para tratamientos en la variable ganancia de peso semanal 1era a 4ta semana

\begin{tabular}{|c|c|c|c|c|c|c|c|c|}
\hline \multirow[t]{2}{*}{ Tratamientos } & \multicolumn{2}{|c|}{ Semana 1} & \multicolumn{2}{|c|}{ Semana 2} & \multicolumn{2}{|c|}{ Semana 3} & \multicolumn{2}{|c|}{ Semana 4} \\
\hline & Media & Rango & Media & Rango & Media & Rango & Media & Rango \\
\hline $\mathrm{T} 2$ & 201.57 & A & 218.86 & A & 244.71 & A & 264.57 & A \\
\hline $\mathrm{T} 1$ & 195.71 & B & 212.00 & B & 239.86 & B & 257.00 & B \\
\hline T0 & 75.8 & $\mathrm{C}$ & 88.00 & $\mathrm{C}$ & 102.00 & $\mathrm{C}$ & 108.60 & C \\
\hline
\end{tabular}

Tabla 4 Análisis de varianza para la variable ganancia de peso: 5ta a 8va semana

\begin{tabular}{|c|c|c|c|c|c|c|c|c|c|}
\hline \multirow[t]{2}{*}{ F de V } & \multirow[t]{2}{*}{ GL } & \multicolumn{2}{|c|}{ Semana 5} & \multicolumn{2}{|c|}{ Semana 6} & \multicolumn{2}{|c|}{ Semana 7} & \multicolumn{2}{|c|}{ Semana 8} \\
\hline & & $\mathbf{C M}$ & $\begin{array}{c}\text { Valor de } \\
\text { F }\end{array}$ & $\mathbf{C M}$ & Valor de F & CM & $\begin{array}{c}\text { Valor de } \\
\text { F }\end{array}$ & $\mathbf{C M}$ & Valor de F \\
\hline Tratamientos & 2 & 44738.97 & $7300.07 * *$ & 47725.38 & $15342.26^{* *}$ & 32723.86 & $61.37 * *$ & 28663.67 & $15767.83^{* *}$ \\
\hline Error & 16 & 6.13 & & 3.11 & & 533.18 & & 1.82 & \\
\hline Total & 18 & \multirow{2}{*}{\multicolumn{2}{|c|}{1.08}} & & & \multirow{2}{*}{\multicolumn{2}{|c|}{11.18}} & \multirow{2}{*}{\multicolumn{2}{|c|}{0.69}} \\
\hline C.V (\%) & & & & \multicolumn{2}{|c|}{0.76} & & & & \\
\hline
\end{tabular}

Tabla 5 Prueba de Tukey al 5\% para tratamientos en la variable ganancia de peso: 5 ta a 8va semana

\begin{tabular}{cccccccccc}
\hline Tratamientos & \multicolumn{2}{c}{ Semana 5 } & \multicolumn{2}{c}{ Semana 6 } & \multicolumn{2}{c}{ Semana 7 } & \multicolumn{2}{c}{ Semana 8 } \\
\cline { 2 - 10 } & Media & Rango & Media & Rango & Media & Rango & \multicolumn{2}{c}{ Media } & Rango \\
T2 & 276.00 & A & 277.43 & A & 252.00 & A & 230.14 & A & B \\
T1 & 265.86 & B & 268.86 & B & 230.43 & A & 224.71 & B \\
T0 & 115.40 & C & 112.40 & C & 109.60 & B & 102.80 & C \\
\hline
\end{tabular}

Para la ganancia de peso de la quinta a octava semana (tabla 4), se reporta el análisis de varianza para la variable ganancia de peso desde la semana cinco a la semana ocho de engorde, se determinó que existe una significación al $1 \%$ para tratamientos, lo que significa que existen diferencias en la ganancia de peso de cada tratamiento. El coeficiente 63 de variación para la quinta semana fue de $1.08 \%$, para la sexta semana de $0.76 \%$, para la séptima semana de $11.18 \%$ y para la octava semana de $0.69 \%$, cuyos valores demuestran una aceptable confiabilidad en los resultado.

Aplicando la prueba de Tukey al 5\%, para la variable ganancia de peso desde la semana cinco a la 
semana ocho (tabla 5); se registran tres rangos de significación en la quinta semana siendo T2 (100\% FVH de avena con solución nutritiva) con una ganancia de peso promedio de $276 \mathrm{~g}$, el segundo rango lo ocupa T1 (100\% FVH de avena sin solución nutritiva) con una ganancia de peso de $265.86 \mathrm{~g}$ y el tercer rango lo ocupa T0 (avena de corte) con una ganancia de peso de $115.40 \mathrm{~g}$. En la sexta semana se registran tres rangos de significación siendo T2 (100\% FVH de avena con solución nutritiva) con una ganancia de peso promedio de 277.43 g, Así mismo para la séptima semana se registran dos rangos de significación, siendo T2 (100\% FVH de avena con solución nutritiva) con una ganancia de peso promedio de $252.00 \mathrm{~g}$, seguido de T1 (100\% FVH de avena sin solución nutritiva) con una ganancia de peso de 230.4 g. En la octava semana se registra de igual forma $\mathrm{T} 2(100 \% \mathrm{FVH}$ de avena con solución nutritiva) con una ganancia de peso promedio de $230.14 \mathrm{~g}$. Con estos resultados se deduce que la utilización del FVH con solución nutritiva produce mayor ganancia de peso semanal.

Figura 1 Representación gráfica de la ganancia de peso de los tratamientos

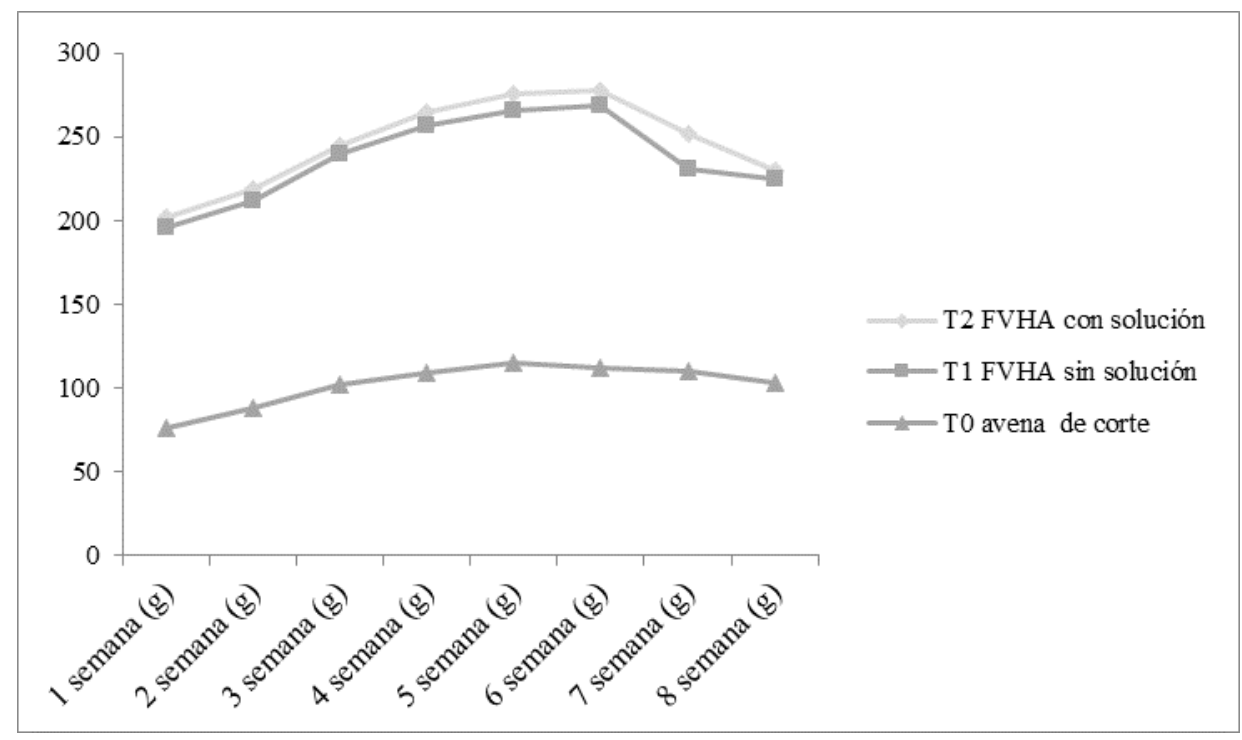

En el (figura 1), se encuentra la curva de crecimiento de la ganancia de peso desde la semana 1 hasta la semana 8 del ensayo de los tres tratamientos, en donde el T0 aumenta ligeramente la ganancia de peso desde la primera semana a la quinta semana, llegando a su máxima ganancia de peso de $115.40 \mathrm{~g}$ a la quinta semana y disminuyendo la ganancia de peso en la sexta semana hasta la octava semana. El T1 y el T2 van ganando peso desde la primera semana hasta la sexta semana con su máxima ganancia de 268.86 g para T1 y 277.43 g para T2 en la sexta semana, en la séptima y octava semana va disminuyendo la ganancia de peso para $\mathrm{T} 1$ y T2. La conversión alimenticia de la primera semana a la cuarta (tabla 6), se reporta el análisis de varianza para la variable conversión alimenticia, desde la semana uno a la semana cuatro con respecto al engorde, donde se determinó que existen diferencias estadísticas significativas al $1 \%$ para tratamientos. El coeficiente de variación para la primera semana fue de $0.28 \%$, para la segunda semana de $3.38 \%$, para la tercera semana de $0.17 \%$ y para la cuarta 
semana de $0.17 \%$, cuyos valores demuestran una aceptable confiabilidad en los resultados.

Aplicando la prueba de Tukey al 5\% para la variable conversión alimenticia, desde la semana uno a la semana cuatro (tabla 7); se registran tres rangos de significación en la primera semana, con la mejor conversión alimenticia el T2 (100\% FVH de avena con solución nutritiva) con conversión promedio de 4.8; el segundo rango lo ocupa T1 (100\% FVH de avena sin solución nutritiva) con una conversión alimenticia promedio de 5.16 y el tercer rango lo ocupa T0 (Avena de corte) con una conversión alimenticia promedio de 12.07. En la segunda semana se registran dos rangos de significación siendo T2
(100\% FVH de avena con solución nutritiva) con una conversión alimenticia promedio de 5. En la tercera semana se registran tres rangos de significación, siendo T2 (100\% FVH de avena con solución nutritiva) con una conversión alimenticia promedio de 4.78. Para la cuarta semana se registraron tres rangos de significación, siendo T2 (100\% FVH de avena con solución nutritiva) con una conversión alimenticia promedio de 4.83, el segundo rango lo ocupa T1 (100\% FVH de avena sin solución nutritiva) con una conversión alimenticia promedio de 5.22. Con estos resultados se deduce que la utilización del FVH con solución nutritiva produce la mejor conversión alimenticia.

Tabla 6 Análisis de varianza para la variable conversión alimenticia: 1era a 4ta semana

\begin{tabular}{|c|c|c|c|c|c|c|c|c|c|}
\hline \multirow{2}{*}{ F de V } & \multirow[t]{2}{*}{ GL } & \multicolumn{2}{|c|}{ Semana 1} & \multicolumn{2}{|c|}{ Semana 2} & \multicolumn{2}{|c|}{ Semana 3} & \multicolumn{2}{|c|}{ Semana 4} \\
\hline & & $\mathrm{CM}$ & Valor de F & $\mathrm{CM}$ & Valor de $\mathrm{F}$ & $\mathrm{CM}$ & Valor de F & $\mathrm{CM}$ & Valor de F \\
\hline Tratamientos & 2 & 92.69 & $244604.94 * *$ & 84.85 & $1538.12^{* *}$ & 73.27 & $571443.84 * *$ & 76.96 & $568559.86^{* * *}$ \\
\hline Error & 16 & 0.00038 & & 0.06 & & 0.00 & & 0.00014 & \\
\hline Total & 18 & & & & & & & & \\
\hline C.V & & & .28 & & 3.38 & & 0.17 & & .17 \\
\hline
\end{tabular}

Tabla 7 Prueba de Tukey al 5\% para tratamientos en la variable conversión alimenticia: 1era a 4ta semana

\begin{tabular}{ccccccccccc}
\hline Tratamientos & \multicolumn{2}{c}{ Semana 1 } & \multicolumn{2}{c}{ Semana 2 } & \multicolumn{2}{c}{ Semana 3 } & \multicolumn{2}{c}{ Semana 4 } \\
\cline { 2 - 11 } T2 & Media & Rango & Media & Rango & Media & Rango & Media & \multicolumn{2}{c}{ Rango } \\
T1 & 4.80 & A & 5.03 & A & 4.78 & A & 4.83 & A & \\
T0 & 5.16 & B & 5.29 & A & 5.11 & B & 5.22 & B \\
\hline
\end{tabular}

Tabla 8 Análisis de varianza para la variable conversión alimenticia: 5 ta a 8va semana

\begin{tabular}{|c|c|c|c|c|c|c|c|c|c|}
\hline \multirow{2}{*}{ F de V } & \multirow[b]{2}{*}{ GL } & \multicolumn{2}{|c|}{ Semana 5} & \multicolumn{2}{|c|}{ Semana 6} & \multicolumn{2}{|c|}{ Semana 7} & \multicolumn{2}{|c|}{ Semana 8} \\
\hline & & $\mathbf{C M}$ & Valor de F & $\mathbf{C M}$ & Valor de F & $\mathbf{C M}$ & Valor de F & $\mathbf{C M}$ & Valor de $F$ \\
\hline Tratamientos & 2 & 80.42 & $319870.36^{* *}$ & 106.48 & $414673.11^{* *}$ & 116.61 & $265667.80 * *$ & 224.03 & $45.28 * *$ \\
\hline Error & 16 & 0.00025 & & 0.00026 & & 0.00044 & & 4.95 & \\
\hline Total & 18 & & & & & & & & \\
\hline C.V & & \multicolumn{2}{|c|}{0.23} & \multicolumn{2}{|c|}{0.21} & \multicolumn{2}{|c|}{0.24} & \multicolumn{2}{|c|}{20.72} \\
\hline
\end{tabular}

Tabla 9 Prueba de Tukey al 5\% para tratamientos en la variable conversión alimenticia: 5 ta a 8va semana

\begin{tabular}{|c|c|c|c|c|c|c|c|c|}
\hline \multirow[t]{2}{*}{ Tratamientos } & \multicolumn{2}{|c|}{ Semana 5} & \multicolumn{2}{|c|}{ Semana 6} & \multicolumn{2}{|c|}{ Semana 7} & \multicolumn{2}{|c|}{ Semana 8} \\
\hline & Media & Rango & Media & Rango & Media & Rango & Media & Rango \\
\hline $\mathbf{T 2}$ & 5.07 & A & 5.51 & A & 6.48 & A & 7.55 & A \\
\hline T1 & 5.48 & B & 5.91 & B & 6.96 & A & 8.13 & A \\
\hline
\end{tabular}


En la (tabla 8), se reporta el análisis de varianza para la variable conversión alimenticia desde la semana cinco a la semana ocho de engorde, se determinó que existen diferencias estadísticamente significativas al $1 \%$ para tratamientos. El coeficiente de variación para la primera semana fue de $0.23 \%$, para la segunda semana de $0.21 \%$, para la tercera semana de $0.24 \%$ y para la cuarta semana de $20.72 \%$, cuyos valores demuestran una aceptable confiabilidad en los resultados.

Aplicando la prueba de Tukey al 5\%, para la variable conversión alimenticia, desde la semana cinco a la semana ocho (tabla 9), se registran tres rangos de significación en la quinta semana, con la mejor conversión alimenticia el T2 (100\% FVH de avena con solución nutritiva) con una conversión promedio de 5.07. En la sexta semana se registran tres rangos de significación siendo el mejor T2 (100\% FVH de avena con solución nutritiva) con una conversión alimenticia promedio de 5.51. Analizando la séptima semana se registran dos rangos de significación, siendo el mejor T2 (100\% FVH de avena con solución nutritiva) con una conversión alimenticia promedio de 6.78. Para la octava semana se registran tres rangos de significación, siendo el mejor T2 (100\% FVH de avena con solución nutritiva) con una conversión alimenticia promedio de 7.55. Con estos resultados se deduce que la utilización del FVH con solución nutritiva produce mejor conversión alimenticia.

Figura 2 Representación gráfica de la conversión alimenticia de los tratamientos

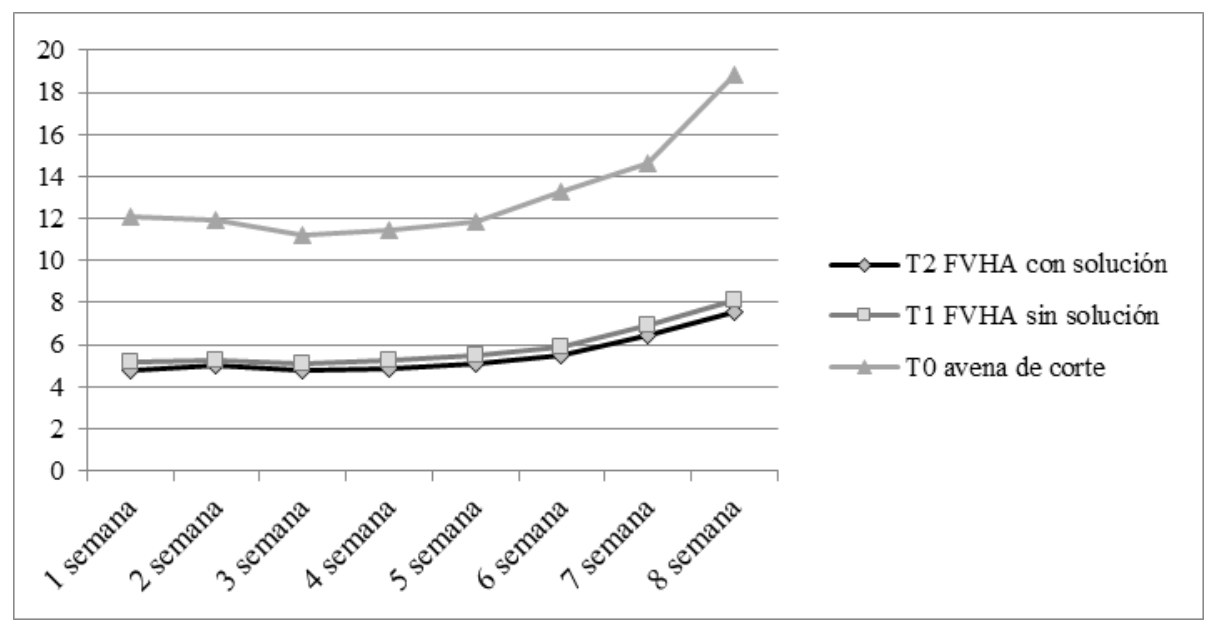

En la (figura 2), se encuentra la curva de crecimiento para la conversión alimenticia desde la semana 1 hasta la semana 8 del ensayo de los tres tratamientos, en la primera y segunda semana el T1 y T2 se aumenta la conversión alimenticia, en la tercera semana disminuye ligeramente, desde la cuarta hasta la octava semana en los T1 y T2 va aumentando la conversión alimenticia hasta llegar a 8.13 para $\mathrm{T} 1$ y 7.55 para T2. El T0 en la primera semana presentó un aumento de la conversión alimenticia, en la se- gunda y tercera semana disminuye ligeramente la conversión, de la cuarta hasta la octava semana hay un mayor aumento de conversión alimenticia ubicándose en 18.85. Esto significa que mientras más alto sea el índice de conversión alimenticia peor son los resultados y mientras más bajo sea el índice de conversión mejores resultados se obtienen. Por lo tanto, el T2 obtuvo la mejor conversión alimenticia promedio con 5.5 seguido del T1 con 5.9 de conversión, debido a que los forrajes hidropónicos poseen 
un alto valor nutritivo y mejor digestibilidad que el T0.

Los resultados del análisis bromatológico del FVHA sin solución nutritiva, FVHA con solución nutritiva y de la avena de corte, efectuado en el Laboratorio de Servicio de Análisis e Investigación en Alimen- tos del Instituto Nacional Autónomo de Investigaciones Agropecuarias (INIAP) de la provincia de Pichincha. Con el objetivo de observar los contenidos de humedad, cenizas, fibra, proteína, grasa y extractos libres de nitrógeno (E.L.N.).

Tabla 10 Análisis bromatológico de tratamientos

\begin{tabular}{lcccccc}
\hline \multicolumn{1}{c}{ Tratamientos } & \multicolumn{5}{c}{ Parámetros Analizados } \\
\cline { 2 - 7 } & Humedad \% & Cenizas \% & Grasa \% & Proteína \% & Fibra \% & E.L.N \% \\
T0 (avena de corte) & 78.87 & 8.14 & 1.71 & 7.20 & 33.26 & 49.69 \\
T1 (FVH de avena sin solución nutritiva & 83.72 & 3.67 & 5.57 & 15.53 & 21.28 & 53.86 \\
T2 (FVH de avena con solución nutritiva & 85.59 & 4.42 & 4.35 & 16.78 & 22.51 & 51.94 \\
\hline INIAP 2015 & & & &
\end{tabular}

Figura 3 Representación gráfica del análisis bromatológico de los tratamientos

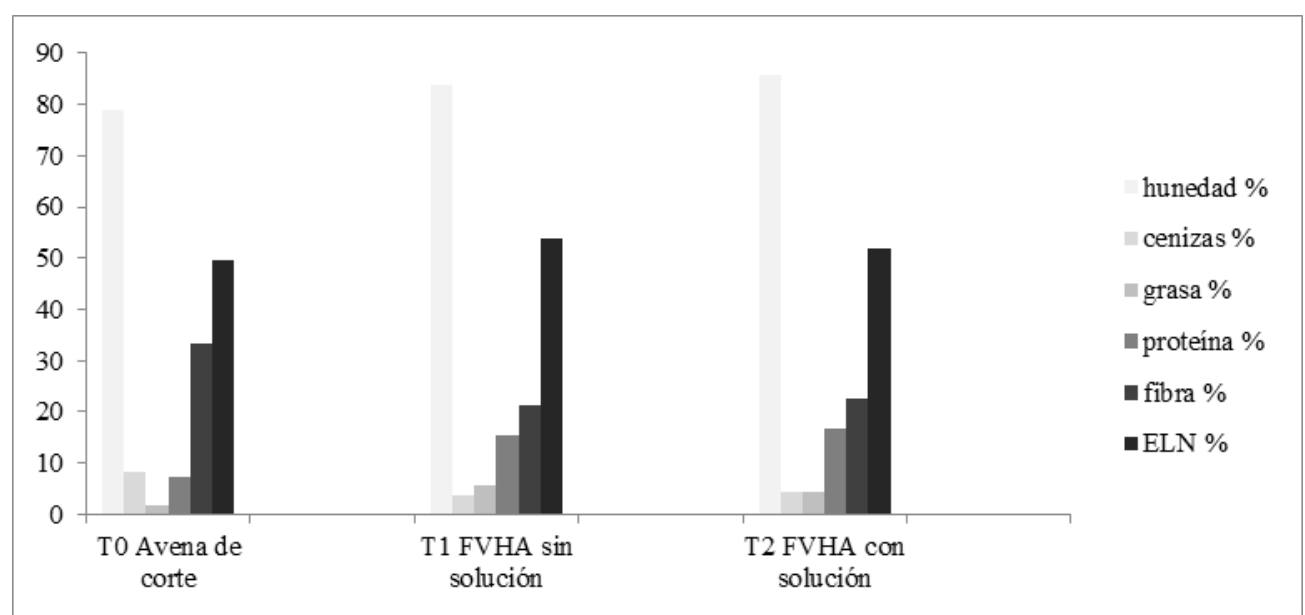

En la (tabla 10, fig 3), se resumen los resultados del análisis bromatológico donde se puede deducir que el T2 (FVH de avena con solución nutritiva) reporta el mayor contenido de humedad con $85.59 \%$, seguido del T1 (FVH de avena sin solución nutritiva) con $83.51 \%$ y el T0 (avena de corte) reporta el menor contenido de humedad con $78.87 \%$. El mejor contenido en cenizas reporta el T0 (avena de corte) con $8.14 \%$ en segundo lugar se encuentra el T2 (FVH de avena con solución nutritiva) con $4.42 \%$ y seguido el T1 (FVH de avena sin solución nutritiva) con 67
3.67\%. El mayor contenido en grasa (E.E.) pertenece al T1 (FVH de avena sin solución nutritiva) con $5.57 \%$ seguido del T2 (FVH de avena con solución nutritiva) con $4.95 \%$ y con menor contenido de grasa (E.E.) se encuentra el T0 (avena de corte) con $1.71 \%$. El mejor contenido de proteína reporta el T2 (FVH de avena con solución nutritiva) con $16.78 \%$ seguido del T1 (FVH de avena sin solución nutritiva) con $15.62 \%$ y el T0 (avena de corte) reportó el contenido más bajo de proteína con $7.20 \%$. El mayor contenido en fibra reportó el T0 (avena de corte) 
con 33.26\% en segundo lugar se presentó el T2 (FVH de avena con solución nutritiva) con $22.51 \%$ seguido por el T1 (FVH de avena sin solución nutritiva) con $21.28 \%$. El mayor contenido de E.L.N. (extractos libres de nitrógeno) perteneció al T1 (FVH de avena sin solución nutritiva) con $53.96 \%$ seguido del T2 (FVH de avena con solución nutritiva) con $51.94 \%$ y en tercer lugar se encontró el T0 (avena de corte) con $49.69 \%$.

Los costos variables del ensayo desglosado por tratamiento, la variación de los costos (tabla 11), está dada básicamente por los diferentes precios de cada alimentación: el precio en la mano de obra, los materiales utilizados, servicios, dotación de alimento y conejos destetados. Deduciendo que el tratamiento con mayor inversión es el T0 (avena de corte) con 58.87 USD, mientras que la menor inversión se encuentra en el T1 (FVH de avena sin solución nutritiva) con 47.71 USD, seguidamente con el T2 (FVH de avena con solución nutritiva) con 47.83 USD, ya que la producción de forraje se lo realizó conjuntamente con la crianza y engorde de los conejos, lo que resultó un ahorro en costos de inversión en la mano de obra, servicios básicos, materiales y transporte, los ingresos del ensayo desglosado por tratamiento. La variación de los ingresos está dada básicamente por el rendimiento y el precio por animal. El tratamiento con mayor ingreso es T1 (FVH de avena con solución nutritiva) con 112 USD, mientras que el menor ingreso reportó el testigo (avena de corte) con 55 USD, éste valor se afectó por la muerte de varios conejos y por los pesos bajos a la venta, los cuales no superaron los $2.2 \mathrm{~kg}$.

Tabla 11 Costos de inversión del ensayo por tratamientos

\begin{tabular}{|c|c|c|c|c|c|c|}
\hline Tratamientos & $\begin{array}{c}\text { Mano de } \\
\text { obra (USD) }\end{array}$ & $\begin{array}{l}\text { Materiales } \\
\text { (USD) }\end{array}$ & $\begin{array}{c}\text { Servicios } \\
\text { (USD) }\end{array}$ & $\begin{array}{c}\text { Dotación de } \\
\text { Alimento (USD) }\end{array}$ & $\begin{array}{l}\text { Conejos } \\
\text { (USD) }\end{array}$ & $\begin{array}{l}\text { Costo total } \\
\text { (USD) }\end{array}$ \\
\hline T0 & 15.00 & 5.91 & 1.00 & 15.96 & 21.00 & 58.87 \\
\hline T1 & 15.00 & 7.61 & 1.00 & 3.10 & 21.00 & 47.71 \\
\hline $\mathbf{T} 2$ & 15.00 & 7.73 & 1.00 & 3.10 & 21.00 & 47.83 \\
\hline
\end{tabular}

Tabla 12 Cálculo de la relación beneficio costo de los tratamientos con tasa de interés al $11 \%$

\begin{tabular}{ccccccc}
\hline Tratamientos & $\begin{array}{c}\text { Ingresos } \\
\text { total }\end{array}$ & $\begin{array}{c}\text { Costos } \\
\text { total }\end{array}$ & $\begin{array}{c}\text { Factor de } \\
\text { actualización }\end{array}$ & $\begin{array}{c}\text { Costo total } \\
\text { actualizado }\end{array}$ & $\begin{array}{c}\text { Ingresos } \\
\text { actualizados }\end{array}$ & $\begin{array}{c}\text { Valor } \\
\text { neto actual }\end{array}$ \\
\hline T0 & 55.00 & 58.87 & 0.98 & 57.69 & 53.90 & -3.79 \\
T1 & 108.50 & 47.71 & 0.98 & 46.76 & 106.33 & 59.07 \\
T2 & 112.00 & 47.83 & 0.98 & 46.87 & 109.76 & 62.89 \\
\hline
\end{tabular}

La (tabla 12), muestra el análisis económico realizado a través del indicador Beneficio/Costo, tomando en consideración los índices de mortalidad y el peso en pie; se determinó que la mayor rentabilidad se consiguió con el T2 (FVH de avena con solución nutritiva) con un beneficio/costo de 1.34 USD, seguido de T1 (FVH de avena sin solución nutritiva) con un beneficio/costo de 1.27 USD, indicando que ambos tratamientos son rentables y en los cuales se obtienen ganancias económicas.

\section{Discusión}

La ganancia de peso semanal de la primera a la cuarta semana (tabla 2), se reporta el análisis de varianza para la variable ganancia de peso desde la semana uno a la semana cuatro de engorde, se de- 
terminó que existen diferencias estadísticas al $1 \%$ para tratamientos en la ganancia de peso de cada tratamiento, con una ganancia de peso promedio semanal de $201.57 \mathrm{~g}$, el segundo rango lo ocupa T1 (100\% FVH de avena sin solución nutritiva) con una ganancia de peso de $195.71 \mathrm{~g}$ y el último rango de significación lo ocupa T0 (Avena de corte) con una ganancia de peso de $75.8 \mathrm{~g}$. En la segunda semana se registran tres rangos de significación siendo el mejor T2 (100\% FVH de avena con solución nutritiva) con una ganancia de peso promedio de $218.86 \mathrm{~g}$, el segundo rango lo ocupa T1 (100\% FVH de avena sin solución nutritiva) con una ganancia de peso de $212.00 \mathrm{~g}$ y el último rango lo ocupa T0 (Avena de corte) con una ganancia de peso de 88.00 g.

Para la ganancia de peso de la quinta semana a la octava, siendo el mejor T2 (100\% FVH de avena con solución nutritiva) con una ganancia de peso promedio de $276 \mathrm{~g}$, el segundo rango lo ocupa T1 (100\% FVH de avena sin solución nutritiva) con una ganancia de peso de $265.86 \mathrm{~g}$ y el tercer rango lo ocupa T0 (Avena de corte) con una ganancia de peso de $115.40 \mathrm{~g}$. En la sexta semana se registran tres rangos de significación siendo el mejor T2 (100\% FVH de avena con solución nutritiva) con una ganancia de peso promedio de $277.43 \mathrm{~g}$, el segundo rango lo ocupa T1 (100\% FVH de avena sin solución nutritiva) con una ganancia de peso de 268.86 g y el ultimo rango lo ocupa T0 (Avena de corte) con una ganancia de peso de $112.40 \mathrm{~g}$. En base a estos resultados concuerdo con (Gonzáles 2004), quienes manifiestan que la ganancia de peso en conejos de engorde varía entre 252 a 266 g promedio semanal. El T2 obtuvo la mejor ganancia de peso promedio semanal con $245.66 \mathrm{~g}$ seguido del T1 con 236.80 g debido a que los forrajes hidropónicos poseen un alto valor nutritivo y favorecen a 69 una mayor velocidad de crecimiento, la peor ganancia de peso obtuvo el T0 con un promedio semanal de 101.82 g debido a que la avena de corte no contiene los requerimientos necesarios para el desarrollo del conejo.

Por otro lado (Moreno 2013). obtuvo una ganancia de peso favorable para T2 (alimento balanceado) $39.26 \mathrm{~g}$, con una desviación estándar de 4.59 por lo que obtuvo mayor consumo de alimento lo que se reflejó en una mayor velocidad de crecimiento terminando su engorda en 35 días, por su parte la ganancia de peso en el T1 (FVHT), fue de $30.32 \mathrm{~g}$, con una desviación estándar de 5.13 en promedio diario que no es mala si consideramos que se alcanzó el peso ideal de sacrificio a los 41 días de engorda que se encuentra dentro de los tiempos normales de engorda en granjas.

Los resultados del análisis bromatológico donde se puede deducir que el T2 (FVH de avena con solución nutritiva) reporta el mayor contenido de humedad con $85.59 \%$, seguido del T1 (FVH de avena sin solución nutritiva) con $83.51 \%$ y el T0 (avena de corte) reporta el menor contenido de humedad con $78.87 \%$. El mejor contenido en cenizas reporta el T0 (avena de corte) con $8.14 \%$ en segundo lugar se encuentra el T2 (FVH de avena con solución nutritiva) con $4.42 \%$ y seguido el T1 (FVH de avena sin solución nutritiva) con $3.67 \%$. El mayor contenido en grasa (E.E.) pertenece al T1 (FVH de avena sin solución nutritiva) con $5.57 \%$ seguido del T2 (FVH de avena con solución nutritiva) con $4.95 \%$ y con menor contenido de grasa (E.E.) se encuentra el T0 (avena de corte) con $1.71 \%$. El mejor contenido de proteína reporta el T2 (FVH de avena con solución nutritiva) con $16.78 \%$ seguido del T1 (FVH de avena sin solución nutritiva) con $15.62 \%$ y el T0 (avena de corte) reportó el contenido más bajo de proteína con $7.20 \%$. El mayor contenido en fibra reportó el 
T0 (avena de corte) con 33.26\% en segundo lugar se presentó el T2 (FVH de avena con solución nutritiva) con $22.51 \%$ seguido por el $\mathrm{T} 1$ (FVH de avena sin solución nutritiva) con $21.28 \%$. El mayor contenido de E.L.N. (extractos libres de nitrógeno) perteneció al T1 (FVH de avena sin solución nutritiva) con 53.96\% seguido del T2 (FVH de avena con solución nutritiva) con $51.94 \%$ y en tercer lugar se encontró el T0 (avena de corte) con 49.69\%.

Según (Rodríguez 1999) indica que los niveles de grasa en la dieta de los conejos pueden fluctuar entre 2 a $5 \%$, puesto que hace más apetitosos los alimentos, facilitan la absorción de las vitaminas liposolubles A, D, E, K y promueven el brillo y lustre del pelo, coincidiendo así con los resultados obtenidos en el ensayo. Los mejores contenidos de proteína pertenecen al $\mathrm{T} 2$ con $16.78 \%$ y $\mathrm{T} 1$ con $15.53 \%$ que son valores que se encuentran dentro de los requerimientos de proteína bruta que necesita el conejo, por lo que coincide con Brenes-Payá et al. (1978) que determina que el requerimiento de proteína bruta en conejos es de 15 a 17\%. De acuerdo con Caravaca-Rodríguez (2005) que explica que una deficiencia proteica durante el cebo provoca una menor formación de músculo y un menor crecimiento; así como lo mencionado por Müller et al. (2005) que pueden haber reducciones en proteína. Según (Gonzáles 2004) el requerimiento en fibra del conejo de cebo es de 12 a $16 \%$ aunque llega hasta el $20 \%$, cuanto más aumenta el nivel de fibra de una ración, más disminuye el de energía aumentando por consecuencia el consumo, esto explica la relación fibra-energía-proteína. Por tanto los resultados de nuestro ensayo del contenido de fibra no están dentro del rango por lo que el nivel de energía fue menor.

\section{Conflictos de intereses}

La investigación se la realizó en la Concepción, perteneciente a la parroquia Atahualpa cantón Ambato y no presenta conflictos de interés.

\section{Agradecimientos}

Los autores agradecen a la Universidad Técnica de Ambato. Facultad de Ciencias Agropecuarias, por el apoyo técnico, científico y logístico realizado a la presente investigación.

\section{Literatura citada}

Brenes-Payá A, Brenes-Payá J, Pontes-Pontes M. Requerimientos nutritivos del conejo. Cunicult 1978;3(13):117-27.

Caravaca-Rodriguez FP. Bases de la producción animal. Universidad de Sevilla; 2005. p. 512.

Carmona FF, Poblete-Pérez EC, Huerta-Pizarro MA. Respuesta productiva de conejos alimentados con forraje verde hidropónico de avena, como reemplazo parcial de concentrado comercial. Acta Agron 2011;60:183-9.

Cerrillo-Soto MA, Juárez-Reyes AS, RiveraAhumada JA, Guerrero-Cervantes M, Ramirez-Lozano RG, Bernal-Barragán H. Producción de biomasa y valor nutricional del forraje verde hidropónico de trigo y avena. Interciencia 2012;37:906-13.

Fuentes F, Poblete C, Huerta M, Palape I. Evaluación de la producción y calidad nutritiva de avena como forraje verde hidropónico en condiciones de desierto. IDESIA 2011;29:75-81.

Gonzáles R. Nutrición y alimentación del conejo. Área Interdisciplinaria de Ciencias Agropecuarias. Universidad Autónoma de Baja California 
del Sur. 2004. 1era edición. Disponible en http://www.uabcs.mx/maestros/descartados/mt o05/index.htm. Consultado el 30 de octubre del 2015.

Morales MA, Fuente B, Juárez M, Ávila E. Effect of substituting hydroponic green barley forage for a commercial feed on performance of growing rabbits. World Rabbit Sci 2009;17:358.

Moreno H. Evaluación de Forraje Verde Hidropónico en la Alimentación de conejos de Engorda. [Tesis de Licenciatura]. Universidad Michoacana de San Nicolás de Hidalgo. México; 2013.

Müller L, Manfron P, Santos O, Medeiros S, Haut V, Dourado D. Produção e composição bromatologica da forragem hidropônica de milho, Zea mays L., com diferentes densidades de semeadura e datas de colheita. Zootecnia Trop 2005;23(2):105-19.

Leandro C. Producción de Forraje Verde Hidropónico; 2010.

Palma OR, Hurtado EA. Comportamiento productivo de conejos durante el período de crecimiento-engorde alimentados con frutos de mango (Mangifera indica) en sustitución parcial del alimento balanceado comercial. Idesia 2010;28(1):33-7.
Rodriguez-Muela C, Rodriguez HE, Ruiz O, Flores A, Grado JA, Arzola C. Use of green fodder produced in hydroponics systems as supplement for salers lactating cows during the dry season. Proc West Sect Am Soc Anim Sci 2005;56:271-4.

Rea H, Mora M. Evaluación de cuatro forrajes hidropónicos en la alimentación de cuyes (Cavia porcellus), durante la fase de crecimiento y engorde en el criadero "El Mirador", barrio Langos San Alfonso del cantón Guano, provincia de Chimborazo. [Tesis de Licenciatura]. Escuela Superior Politécnica de Chimborazo. Facultad de Ciencias Agrarias; 2012. p. 121.

Rodríguez H. 1999. Nutrición de los conejos. 1st ed. Colegio de Ciencias Agropecuarias. Puerto Rico. Disponible en. www.uprm.edu/agricultura/ sea/publicaciones/Nutriciondelosconejos.pdf.

Samperio G. Hidroponía Comercial. Editorial DIANA S.A. Primera edición. México; 1999. p. 15-7. 